\title{
1 Atria: An Ultra-fast and Accurate Trimmer for Adapter and 2 Quality Trimming
}

4 Jiacheng Chuan ${ }^{1,2}$, Aiguo Zhou ${ }^{1,3}$, Lawrence Richard Hale ${ }^{2}$, Miao $\mathrm{He}^{4}$, Xiang $\mathrm{Li}^{1,{ }^{*}}$

$6 \quad{ }^{1}$ Canadian Food Inspection Agency, Charlottetown, PE C1A5T1, Canada

$7{ }^{2}$ Department of Biology, University of Prince Edward Island, Charlottetown, PE

8 C1A4P3, Canada

$9 \quad{ }^{3}$ Guangdong Laboratory for Lingnan Modern Agriculture, South China Agricultural

10 University, Guangzhou 510642, China

$11{ }^{4}$ School of Life Sciences, Sun Yat-sen University, Guangzhou, Guangdong, 510275,

12 China

13

14 E-mail:

15 jiacheng.chuan@inspection.gc.ca (Chuan J)

16 aiguozhou@scau.edu.cn (Zhou A)

17 lhale@upei.ca (Hale L)

18 lsshem@mail.sysu.edu.cn (He M)

19 sean.li@inspection.gc.ca (Li X)

20 * Corresponding author: Xiang Li, sean.li@inspection.gc.ca

21 Abstract 
22 Background: As Next Generation Sequencing takes a dominant role in terms of output

23 capacity and sequence length, adapters attached to the reads and low-quality bases

24 hinder the performance of downstream analysis directly and implicitly, such as

25 producing false-positive single nucleotide polymorphisms (SNP), and generating

26 fragmented assemblies. A fast trimming algorithm is in demand to remove adapters

27 precisely, especially in read tails with relatively low quality.

28 Findings: We present a trimming program named Atria. Atria matches the adapters in

29 paired reads and finds possible overlapped regions with a super-fast and carefully

30 designed byte-based matching algorithm $(O(n)$ time with $O(1)$ space). Atria also

31 implements multi-threading in both sequence processing and file compression and

32 supports single-end reads.

33 Conclusions: Atria performs favorably in various trimming and runtime benchmarks

34 of both simulated and real data with other cutting-edge trimmers. We also provide an

35 ultra-fast and lightweight byte-based matching algorithm. The algorithm can be used in

36 a broad range of short-sequence matching applications, such as primer search and seed

37 scanning before alignment.

38 Availability \& Implementation: The Atria executables, source code, and benchmark

39 scripts are available at https://github.com/cihga39871/Atria under the MIT license.

41 Research Area: Software and Workflows

42 Classifications: Bioinformatics, Software Engineering 


\section{Statement of Need}

45

\section{Background}

Next generation sequencing (NGS) is a revolutionary new technology that produces massive, high-resolution genome sequence data to facilitate a broad range of biological applications. Illumina paired-end sequencing can read a DNA fragment from both ends and generate accurate reads for downstream bioinformatics analysis, such as assembly, resequencing, transcriptome profiling, variant calling, epigenome profiling, chromatin interaction, and chromosomal rearrangements [1, 2].

In paired-end library preparation, adapter sequences are the technical sequences ligated to both sides of inserts, which are the DNA fragments of interest. Then, DNA molecules with adapters are sequenced from both ends of the inserts so paired-end reads are generated. If insert sizes of paired-end reads are less than the read lengths, inserts are reversely complementary, and adapters are sequenced after reading through the inserts (Fig. 1). Thus, adapter contamination in the 3' end needs to be removed before downstream analysis.

Cleaning adapters can therefore be achieved by searching adapter sequences and/or aligning paired reads (Fig. 1). To date, some trimmers, such as AdapterRemoval [3], Trim Galore [4], and Trimmomatic [5], use both types of information to clean adapters. However, when the quality of sequencing reads decreases, the trimming process employing both types of information is likely to give different trimming suggestions. 
64 Trimmers thus face a bottleneck when working on trimming adapters at accurate

65 positions. Also, extremely short adapters at the low-quality 3' end are sometimes

66 difficult to detect. Thus, trade-offs between trimming truncated adapters, and retaining

67 inserts intact, become necessary.

68 These two issues hinder trimmers from cleaning adapter sequences and leaving

69 DNA inserts intact. To combat this, we launch Atria, an integrated trimming program

70 for NGS data. Atria uses a super-fast byte-based matching algorithm to detect adapters

71 and reverse complementary regions of paired reads, and integrates carefully designed

72 decision rules to infer true adapter positions. Thus, Atria can trim extremely short

73 adapter sequences at accurate positions and not over-trim reads without adapters (Fig.

74 1).

75 In addition to adapter trimming, Atria integrated a set of trimming and filtering

76 methods, such as consensus calling for overlapped regions, quality trimming,

77 homopolymer trimming, $\mathrm{N}$ trimming, hard clipping from both ends, and read 78 complexity filtration.

\section{Implementation}

81 The adapter finding algorithms used in Atria can be categorized in the following

82 portions: DNA encoding, matching algorithm, matching and scoring, decision rules,

83 consensus calling, quality trimming, and IO optimization (Fig. 2). 
85

86

87

88

89

90

91

92

93

94

95

96

97

98

99

100

101

102

103

\section{DNA encoding}

The DNA encoding algorithm is developed based on BioSequences, a Julia package from BioJulia [6]. The original BioSequences package encodes DNA bases A, C, G, T as four-bit codes 0001, 0010, 0100, 1000, respectively. Extended codes are also supported, such as N (1111), S (0110), and gap (0000). DNA sequences are encoded and stored in a contiguous block of Random-Access Memory (RAM) as a dense array of unsigned 64-bit integers (UInt64) (Fig. 2A).

Atria makes use of the property of dense arrays to extract sequences as unsigned integers from available memory locations. When accessing the last several indices of a sequence, the extraction is illegal because operating systems do not allow the loading of data outside of sequence boundary. To solve the issue, Atria constructs a bit-safe sequence array, which elongates the sequence boundary by appending a UInt64 to the end of the original array, and setting all bits after the end of encoded DNA to 0 (Fig. 2A).

It is noticeable that the smallest addressable unit of memory is one byte ( 8 bits) while each DNA is encoded in four bits, so only the even indices of sequence can be directly extracted (defining indices start from 0) (Fig. 2A). The extraction from odd indices requires extra operations, which is avoidable in many scenarios of a welldesigned algorithm. 


\section{Matching algorithm}

109 Given two sequences $a$ and $b$, we plan to match the 16-base-long head of $a$ to each

110 index of $b$. However, only the even indices of $b$ can be extracted from memory without

111 bitwise operations, so we prepared two UInt64 of $a$ : $a_{0}$ and $a_{\text {-. }} a_{0}$ is the 16-mer UInt64

112 loaded from the position 0 of $a$, and $a$ - can be computed from the following bitwise

113 operations: ( $\left.a_{0}>>4 \mid a_{1}<<4\right)$. In this way, $a_{0}$ represents the subsequence of $a$ indexed

114 from 0 to 15 (a[0:15]), and $a$ - represents $a[1: 16]$ (Fig. 2B).

115 In this way, the problem of matching the 16-base-long head of $a$ to each index of $b$

116 is converted to the problem of matching two 16-mers, $a_{0}$, and $a_{\text {-, }}$ to each addressable

117 memory position of $b$. The latter requires less bitwise operations.

118 The number of mismatches $K$ is computed in the formula:

$$
K_{a n, b n}=16-\text { count_ones }(\text { an \& bn) }
$$

120 where count_ones counts the number of ones in the binary representation of the UInt64.

121 Let $k$ denote the user-defined number of mismatches allowed in the 16-mer

122 comparison of UInt64 $a_{n}$ and $b_{n}$ ( $k=2$ by default). After matching $a_{0}$ and $a$ - to each

123 addressable memory position of $b$, if the minimum number of mismatches is not greater

124 than $k$, the smallest index of $b$ of the minimum mismatches is reported. 

scoring, and decision rules.

133 We implement four pairs of matching to utilize properties of paired-end reads

134 thoroughly: (1) matching adapter 1 head to read 1, (2) matching adapter 2 head to read

135 2, (3) matching read 1 head to reverse complement of read 2 and (4) matching read 2

136 head to reverse complement of read 1 (Fig. 2C). If the maximum number of bases

137 matched of (1) and (2) is less than a user-defined cut-off (default is 9), (3) and (4) will

138 be performed with a loosed $k\left(=k_{\text {original }}+1\right)$. If the largest number of matched bases of

139 the four matches is greater than the cut-off, and some matches do not meet the

140 requirement, we will re-run those matches with a loosed $k\left(=k_{\text {original }}+3\right)$ at the insert

141 size indicated from the best match. If the new number of matched bases is greater than

142 the cut-off, the old match will be discarded.

143 The scoring system measures the matching reliability of the whole 16-mer rather

144 than each base. The Phred quality score $Q$ of each base is converted to the probability

$145 P$ of that the corresponding base being correct using the formula: 


$$
P=1-10^{\left(-\frac{Q}{10}\right)}
$$

147 Then, the average base quality $\bar{P}$ of 16 -mer sub-sequence $a$ at the memory position

$148 \quad n$ is computed:

$$
\overline{P_{a_{n}}}=\frac{1}{16} \sum_{i=2 n}^{2 n+15} P_{a[i]}
$$

Notably, if the read quality is too low, it would imply an invalid match. However,

151 in reality, invalid matches are filtered out by the kmer-based algorithm. To solve the

152 discordance, we limit the lower bound of $\bar{P}$ to 0.75 manually.

$$
S_{a_{n}, b_{m}}=\text { count_ones }\left(a_{n} \& b_{m}\right) \cdot \overline{P_{a_{n}}} \cdot \overline{P_{b_{m}}}
$$

155 where count_ones counts the number of ones in the binary representation of the UInt64.

156 When sequence $a$ is a user-defined adapter, $\bar{P}_{a}=1$ is used. Generally, the matching 157 score $S$ is ranged from 0 to 16. 

matching score of the read is the sum of adapter and paired-end matching scores. Then, the matches of the two paired-end reads are compared using the same strategy. If one

for matches with any nmatch $<9$

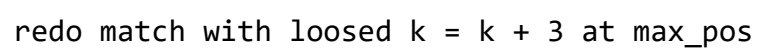

\section{Decision rules}

This module infers correct adapter positions from the four pairs of matching described in the previous section. It is illustrated and self-explanatory in Fig. 2D. First, in each read, the adapter and paired-end matches are compared. The one with the higher matching score is chosen. If both matches support the same adapter position, the read finds an ideal adapter (matching score $>10$ by default) while the other read is too 
194 short to check or the average base accuracy of its 16-mer is less than 0.6 (Phred Q < 5),

195 both reads will be trimmed. If the matching score of a given read pair is less than 10

196 (by default), the read pair will not be trimmed.

197 Other read pairs will be taken a further examination to reduce false positives, which

198 are usually adapter matches at read tails. A read tail is defined as the last several bases

199 (default is 12 bp) of each read. Reads are not trimmed if both statements are true: (1)

200 In any paired read, the adapter is found at the tail, but the paired-end match is not; (2)

201 In both paired reads, adapter and pair-end matches suggest different trimming positions.

202 Before the final trimming, one additional step is required for the accurate

203 positioning of adapter sequences. The previous steps usually assume the read 1 and 2

204 have the same length of insert sizes, but indel in reads usually lead to over or under trim

205 one base. To prevent this circumstance, Atria re-positions the adapter by matching one

206 adjacent base with the first four bp of adapter sequences. The position of the highest

207 number of bases matched is chosen to trim. This step is ignored when the inferred insert

208 size is greater than the read length minus three because, in this situation, the adapter

209 sequence is too short to check.

211 Pseudocode 2: Decision rules

212 function correct_insert_size(pos1, score1, pos2, score2)

213 if $\operatorname{pos} 1==\operatorname{pos} 2$

214 return pos1, score1 + score2

$215 \quad$ else

216 score $=\max ($ score 1, score 2$)$ 
r2_pos, r2_score $=$ correct_insert_size(r2_pos_adpt, r2_score_adpt, r2_pos_pe, 
is_false_positive $=E \& ! R$

246

if r12_score > trim_score \& ! is_false_positive

248

@label “trim”

249

r1_pos_adjusted $=$ adjacent_one_bp_check $(r 1$, adapter1, r12_pos $)$

250

r2_pos_adjusted = adjacent_one_bp_check(r2, adapter2, r12_pos)

251

252

$\operatorname{trim}\left(r 1, r 1 \_p o s \_a d j u s t e d\right)$

255 In this module, the overlapped base pairs of read 1 and 2 are corrected to the

256 corresponding bases with higher quality scores. It has three steps, prediction, 257 assessment, and correction.

258 In the prediction step, Atria makes a preliminary estimate of whether a read pair

259 contains an overlapped region. If adapters are trimmed and the remaining lengths of

260 read 1 and 2 are the same, the prediction passes. If no adapter can be trimmed, two

261 additional matching and scoring are required. The head of the reverse complement of

262 read 2 is matched to read 1 , and the head of the reverse complement of read 1 is matched

263 to read 2. If the two matches reach a consensus, the prediction passes. Otherwise, the 264 prediction fails and consensus calling is skipped.

265 In the assessment step, Atria compares the whole overlapped region using a similar 266 matching algorithm, except that ambiguous bases $(\mathrm{N}, 1111)$ are converted to gaps $(0000)$ 
267 before matching. If the ratio of mismatch is greater than a user-defined value (28\% by

268 default), the assessment fails, and consensus calling is skipped.

269 In the correction step, each base pair in the overlapped region is corrected to the

270 corresponding base with the highest quality score.

272 Quality trimming

273 Atria implements a traditional sliding window algorithm to remove the low-quality tail.

274 The sliding window scans from the front of the read and computes the average Phred

275 quality score of the sliding window. If the average quality is less than a given threshold,

276 the read tail is removed.

278 IO optimization

279 Atria spends more time on reading and writing than matching and trimming, so the key

280 to reducing runtime is to optimize IO usage. Considering that a large amount of RAM

281 is easily accessible nowadays, Atria trades increased RAM usage with decreased time.

282 A large block of memory is allocated for reading input files, which is then wrapped and

283 encoded to FASTQ objects parallelly using multi-threading. On the contrary, in the

284 writing process, Atria unboxes and decodes FASTQ objects to string vectors in parallel

285 and writes sequentially to files. In addition, pigz (parallel gzip) and pbzip2 (parallel

286 bzip2) are called for compression and decompression when needed [7, 8]. Atria also

287 support running with a single thread. 


\section{Comparison to related work}

\section{The performance of adapter trimming on a simulated dataset}

291 We simulated 8.9 G bases with 100 bp paired-end reads from the Arabidopsis thaliana 292 reference genome using the Skewer modified ART, a public NGS read simulator to 293 allow adapters in the reads [9, 10]. The simulation profile was trained from a $101 \mathrm{bp}$ 294 paired-end public dataset SRR330569, and the 33 bp adapter pair used in read 295 simulation is AGATCGGAAGAGCACACGTCTGAACTCCAGTCA and AGATCGGAAGAGCGTCGTGTAGGGAAAGAGTGT [11].

Atria v3.0.0 was benchmarked with cutting-edge and popular trimmers, including

AdapterRemoval v2.3.1 [3], Skewer v0.2.2 [10], Fastp v0.21.0 [12], Ktrim v1.2.1 [13],

Atropos v1.1.29 [14], SeqPurge v2012_12 [15], Trim Galore v0.6.5 [4] and

300 Trimmomatic v0.39 [5]. Only adapter trimming was used, and other trimming and

301 filtration were disabled. Detailed command line arguments are listed in Table S1. Each

302 trimming software was running on an idle Ubuntu 19.10 server with a 32-thread Intel

303 i9-9960X Central Processing Unit (CPU) @ 3.10 GHz, 128 gigabyte (GB) DDR4-3200

304 RAM, and a 2 terabyte (TB) Samsung 970 EVO Solid State Drive (SSD) (sequential 305 reads and writes up to 3.5 and $2.5 \mathrm{~TB} / \mathrm{s})$.

306 The trimming performance was evaluated based on the following metrics: positive 307 predictive value (PPV), as the fraction of the number of correctly trimmed reads to all 
309 the reads with adapters; specificity, as the fraction of the number of untrimmed reads

310 without adapters to all reads without adapters; and Matthew's correlation coefficient

311 (MCC) measuring overall quality of pattern recognition, as

$$
M C C=\frac{T P \cdot T N-F P \cdot F N}{\sqrt{(T P+F P)(T P+F N)(T N+F N)(T N+F P)}}
$$

313 where TP is the number of reads trimmed correctly, TN is the number of untrimmed

314 reads without adapters, FP is the number of over-trimmed reads, and FN is the number

315 of under-trimmed reads $[3,10]$.

Table 1 Adapter trimming performance on the $8.9 \mathrm{G}$ bases with 100 bp paired-

\section{end simulated data}

\begin{tabular}{lllll}
\hline Trimmer & PPV (\%) & Sensitivity (\%) & Specificity (\%) & MCC (\%) \\
\hline Atria & 99.35 & 99.81 & 99.82 & 99.51 \\
AdapterRemoval & 99.42 & 99.94 & 99.83 & 99.61 \\
Atropos & 99.57 & 97.34 & 99.88 & 98.00 \\
Fastp & 98.73 & 99.58 & 99.61 & 98.92 \\
Ktrim & 91.51 & 85.85 & 98.84 & 87.84 \\
SeqPurge & 57.92 & 99.80 & 76.84 & 66.62 \\
Skewer & 99.58 & 99.53 & 99.88 & 99.44 \\
Trim Galore & 40.05 & 82.98 & 62.39 & 38.96 \\
Trimmomatic & 99.29 & 57.86 & 99.88 & 71.05 \\
\hline
\end{tabular}

321 and Skewer were the top-class adapter trimmers in terms of MCC (99.61\%, 99.51\%,

322 99.44\%, respectively) (Table 1). Fastp (98.92\%) and Atropos (98.00\%) were in the

323 second tier (Table 1). Ktrim obtained a good specificity (98.84\%) but sacrificed its 
324 sensitivity (85.85\%), and Trimmomatic achieved an exceptional specificity (99.88\%)

325 by trading off its sensitivity (57.86\%) (Table 1).

326 To compare speed and efficiency, elapsed time (wall time) and average CPU

327 consumption of each trimmer were recorded in different threading (1-32 threads) for

328 uncompressed and gzip compressed data formats (Fig. 3, Table S1). Efficiency was

329 defined as the fraction of processing speed to the percent of CPU utilized, so it was a

330 better measurement, especially in CPU-intensive scenarios, such as running on a server

331 with a job scheduling system or trimming multiple samples at the same time. Ktrim and

332 Atria were two of the fastest trimmers in terms of speed and efficiency, from one to 16

333 threads (Fig. 3, Table S1). For uncompressed data, Trimmomatic was faster than Atria

334 using 8-32 threads, but its real CPU usage was much greater than Atria (Fig. 3, Table

335 S1). The speed and efficiency of AdapterRemoval and Skewer were generally 2-4 times

336 less than Atria, and Atropos was the slowest one (Fig. 3, Table S1). SeqPurge did not

337 support the output of uncompressed data, so it was only tested in the compressed

338 benchmark.

339 When trimming compressed data, the speed of AdapterRemoval, Skewer, Fastp,

340 Atropos and Trimmomatic kept constant when the number of threads increased from 4

341 to 32, because they failed to utilize more than four CPU in the IO process, while Atria

342 and Trim Galore did not have the limitation (Fig. 3, Table S1). Atria was faster than

343 Trim Galore, and the efficiency of Atria was constantly two to three times greater than

344 Trim Galore (Fig. 3, Table S1). SeqPurge showed strange speed curves; when 
348 Atria was the fastest trimmer when trimming compressed files.

\section{The detailed statistics of adapter trimming accuracy on a simulated dataset}

351 The previous portion benchmarks on a whole dataset. This section evaluates trimming accuracy regarding different read properties, including adapter presence or absence, toolkit for read simulation and trimming analysis. original DNA fragment (insert) with a given original insert size is simulated base by

357 base. Adenine, thymine, cytosine, and guanine are randomly chosen repetitively. Then,

358 the insert and adapter sequences are copied base by base with an error profile, which

359 simulates the procedure of sequencing by synthesis. The error profile defines 360 substitution rate, insertion rate, and deletion rate.

361 Twenty-one million read pairs were simulated with a uniform read length (100 bp),

362 different error profiles, adapter length, and original insert sizes. The baseline error 363 profile comprises a $0.1 \%$ substitution rate, $0.001 \%$ insertion rate, and $0.001 \%$ deletion 364 rate, inspired by an Illumina error profile analysis [16]. 1x, 2x, 3x, 4x, and 5x baseline 
chosen. In this way, the reads with the least insert size have full lengths of adapters.

Fig. 4 B illustrates the trimming accuracy on different read error profiles. When adapters are present, the accuracy of all trimmers drops as error rates increase (Fig. 4 
403 inputs were gzip-compressed and trimmed with eight threads, and outputs were also

404 gzip-compressed to reduce massive disk use. All tested trimmers worked in the scenario 405 except that Ktrim could not output gzip files (Table 2).

406 Atria was the fastest program to process and output compressed data in terms of 407 wall time (Table 2). It also achieved the highest number of reads mapped and paired, 
408 and the percent of properly paired reads with or without quality trimming. Generally,

409 higher base mapped is accompanied with higher error rate in the mapping process, so

410 it is important to interpret the two metrics together. Atria had the lowest mapping error

411 rate of $8.1833 \%$ and the forth highest base mapped (Table 2). The trimmers

412 (AdapterRemoval, Fastp, and Atropos) with the highest three error rates has the highest

413 base mapped (Table 2). Our program generally improved more than 5\% compared to

414 other trimmers for the data without quality trimming (Table 2). The mapping statistics

415 of data without quality trimming were generally worse than with quality trimming

416 except for Atria. Specifically, the properly paired rates of other trimmers without

417 quality trimming were 0.5 to $4 \%$ less than with quality trimming (Table 2). Quality

418 trimming also increased the number of mapped and paired reads and reduced the

419 number of unmapped reads (Table 2).

420

421 Table 2 Performance of trimmers on real data 


\begin{tabular}{|c|c|c|c|c|c|c|c|c|c|c|}
\hline \multirow[b]{2}{*}{ Metric } & \multicolumn{2}{|c|}{ Trimming and consensus } & \multicolumn{8}{|c|}{ Trimming only } \\
\hline & Atria & Skewer & Atria & $\mathrm{AR}$ & Atropos & Fastp & Ktrim $^{*}$ & SeqPurge & Trim Galore & Trimmomatic \\
\hline \multicolumn{11}{|c|}{ Low-quality dataset (SRR330569, RNA, Hisat2 mapping) } \\
\hline Elapsed time $(\min : s e c)^{*}$ & 2:38 & 9:19 & 2:32 & 11:29 & 10:08 & 9:17 & $1: 34+\mathrm{GZ}$ & 3:53 & 3:39 & 9:38 \\
\hline \multicolumn{11}{|l|}{ No quality trimming } \\
\hline Reads mapped and paired & $26,126,804$ & $24,694,330$ & $25,781,268$ & $\underline{24.559 .060}$ & $24,505,656$ & $24,545,646$ & $24,196,658$ & $24,240,072$ & $24,046,542$ & $22,797,620$ \\
\hline Reads unmapped & $27,276,761$ & $28,254,804$ & $27,379,299$ & $28,338,455$ & $28,410,022$ & $\underline{28.350 .294}$ & $28,747,248$ & $28,591,442$ & $28,647,873$ & $29,649,287$ \\
\hline Properly paired reads (\%) & 48.3 & 45.6 & 47.6 & 45.3 & 45.2 & 45.3 & 44.5 & 44.7 & 44.2 & 38.3 \\
\hline Base mapped (cigar) & $2,387,212,225$ & $2,354,164,041$ & $2,322,436,545$ & $2,346,791,204$ & $2,341,355,822$ & $\underline{2.344 .847 .438}$ & $2,316,915,673$ & $2,304,776,514$ & $2,317,321,510$ & $2,237,846,534$ \\
\hline Error rate (\%o) & 7.3952 & 9.5897 & 8.1833 & 9.8902 & 9.8536 & 9.8683 & 9.7920 & 9.7904 & 9.6994 & 9.3106 \\
\hline \multicolumn{11}{|l|}{ With quality trimming } \\
\hline Reads mapped and paired & $25,942,092$ & $25,787,464$ & $25,728,206$ & $25,721,788$ & $25,714,956$ & $\underline{25.725 .480}$ & $25,473,670$ & $25,364,392$ & $25,654,498$ & $24,744,754$ \\
\hline Reads unmapped & $27,245,720$ & $27,364,827$ & $27,361,655$ & $27,369,773$ & $27,373,854$ & $\underline{27.360 .527}$ & $27,556,820$ & $27,736,292$ & $27,400,932$ & $28,064,739$ \\
\hline Properly paired reads (\%) & 47.9 & 47.6 & 47.5 & 47.5 & 47.5 & 47.5 & 46.9 & 46.8 & 47.3 & 42.3 \\
\hline Base mapped (cigar) & $2,317,238,536$ & $2,316,981,456$ & $2,302,740,463$ & $2,304,584,269$ & $2,304,325,743$ & 2.304 .437 .244 & $2,292,034,762$ & $2,263,465,110$ & $2,297,815,439$ & $2,246,076,618$ \\
\hline Error rate (\%) & 7.1114 & 7.7882 & 7.8902 & 7.9160 & 7.9141 & 7.9149 & 7.9059 & 7.8787 & 7.8921 & 7.5649 \\
\hline \multicolumn{11}{|c|}{ High-quality dataset (ERR4695159, cell-free DNA, Bowtie2 mapping) } \\
\hline Elapsed time (min:sec)* ${ }^{*}$ & 3:08 & 11:34 & 3:03 & $13: 48$ & 13:41 & 11:29 & $1: 41+\mathrm{GZ}$ & 4:05 & 4:34 & 11:44 \\
\hline \multicolumn{11}{|l|}{ No quality trimming } \\
\hline Reads mapped and paired & $54,367,548$ & $54,287,616$ & $\underline{54.324 .964}$ & $54,319,438$ & $54,299,922$ & $54,322,088$ & $53,087,420$ & $54,446,104$ & $54,218,344$ & $54,128,760$ \\
\hline Reads unmapped & $1,094,145$ & $1,016,244$ & $1,119,103$ & $\underline{989.335}$ & $1,002,745$ & 978,665 & $2,317,968$ & 999,099 & $1,041,005$ & $1,094,752$ \\
\hline Properly paired reads (\%) & 96.8 & 96.7 & $\underline{96.7}$ & $\underline{96.7}$ & 96.7 & 96.7 & 94.1 & 97.0 & 96.4 & 88.6 \\
\hline Base mapped (cigar) & $7,703,820,585$ & $7,700,134,673$ & $7,700,298,217$ & 7.701.482.302 & $7,700,749,164$ & $7,699,298,008$ & $7,607,799,306$ & $7,512,839,360$ & $7,677,087,845$ & $7,720,352,493$ \\
\hline Error rate (\%) & 3.3082 & 3.8388 & 3.8724 & 3.8771 & 3.8834 & $\underline{3.8564}$ & 4.3239 & 3.8007 & 3.9173 & 6.1984 \\
\hline \multicolumn{11}{|l|}{ With quality trimming } \\
\hline Reads mapped and paired & $54,553,566$ & $54,526,276$ & $54,546,192$ & $54,541,948$ & $54,539,502$ & $\underline{54.549 .462}$ & $53,335,674$ & $54,608,308$ & $54,482,002$ & $54,403,982$ \\
\hline Reads unmapped & 965,447 & 984,845 & 967,917 & 970,869 & 973,217 & 826,424 & $2,136,081$ & $\underline{890.884}$ & 999,918 & 914,003 \\
\hline Properly paired reads (\%) & 97.0 & 97.0 & $\underline{97.0}$ & $\underline{97.0}$ & $\underline{97.0}$ & 97.0 & 94.4 & 97.1 & 96.8 & 89.0 \\
\hline Base mapped (cigar) & $7,653,879,312$ & $7,649,380,218$ & $7,646,989,362$ & $7,647,893,624$ & 7.648.184.196 & $7,646,574,606$ & $7,556,468,882$ & $7,461,588,482$ & $7,625,484,706$ & $7,668,777,971$ \\
\hline Error rate (\%o) & 2.9547 & 3.2535 & 3.2678 & 3.2698 & 3.2792 & $\underline{3.2634}$ & 3.7183 & 3.2117 & 3.3109 & 5.5798 \\
\hline
\end{tabular}

423 Note: $\mathrm{AR}$ = AdapterRemoval. In the trimming-only benchmark, bold and underline

424 formats indicate the first and second trimmers (including tie) in terms of each metric, 425 respectively. *Elapsed time (wall time) is benchmarked based on trimming and output 426 gzip files with 8 threads, except that Ktrim cannot output gzip files (marked with time $427+\mathrm{GZ})$.

430 Generally, plasma cell-free DNA is short in length [20], and trimming is extremely 431 important in medical diagnosis. Here, we chose a human genome-wide cell-free DNA 432 dataset ERR4695159. It has $8.4 \mathrm{G}$ bases with 2 x 150 bp read length with 33 bp adapter 433 sequences AGATCGGAAGAGCACACGTCTGAACTCCAGTCA in read 1 and 434 AGATCGGAAGAGCGTCGTGTAGGGAAAGAGTGT in read 2. The benchmark 435 workflow was the same as the RNA-Seq analysis, except that the clean reads were 
436

437

438

450

451

452

453

mapped to the human reference genome hg38 (GRCh38.p13) using Bowtie2 v2.3.5.1 [21].

Atria was also the fastest trimmer in the scenario (3 min 3 s) (Table 2). SeqPurge and Trim Galore finished the task in more than 4 minutes, while others spent more than 11 minutes (Table 2).

In adapter-trimming-only statistics, SeqPurge had the highest mapped and paired reads $(54,446,104)$ and the highest properly paired reads (97.0\%) (Table 2). Atria followed with 54,324,964 mapped and paired reads. Atria, AdapterRemoval, Atropos, and Fastp all had 96.7\% properly paired reads (Table 2).

With quality trimming, the overall performance increased, and properly paired reads were closer; SeqPurge had $97.1 \%$ properly paired reads, with Atria, AdapterRemoval, Atropos, and Fastp close behind at 97.0\% (Table 2). Only 89.0\% of reads were properly paired with Trimmomatic (Table 2).

\section{Discussion}

Atria performs favorably with other cutting-edge adapter trimmers in accuracy, robustness, speed, and efficiency. Its performance is ascribed to the byte-based matching algorithm. The design concept of the algorithm is to minimize any unnecessary CPU operations by taking advantage of the data structure of dense arrays. Matrix-based algorithms, such as the Needleman-Wunsch algorithm and the SmithWaterman algorithm, allocate and update a matrix and perform base-to-base 
comparison [22, 23]. They report every mismatch and gap between two sequences while Atria skips this step since it is focussed on the start positions of successful matches. Despite that, the matching algorithm used in Atria is able to identify mismatch loci when needed.

The byte-based matching algorithm is lightweight and designed for short sequence scanning. Each DNA is encoded in four bits and stores continuously in RAM. A subsequence can be extracted as an unsigned integer from a given memory position. For example, a 64-bit unsigned integer (UInt) represents a 16-mer, and a 128-bit UInt represents a 32-mer. The comparison between two sub-sequences is completed within the accumulator register, a CPU unit for arithmetic or logical operation. It does not require addressing or updating a scoring matrix from RAM. When comparing a short sequence, such as an adapter, to a long sequence, such as the read, the 16-mer of the short sequence is compared to every position of the long sequence. Hence, the bytebased matching algorithm has $O(n)$ expected time complexity and $O(1)$ space complexity in adapter matching, where $\mathrm{n}$ is the length of the long sequence.

The algorithm also has its limitations. It only reports the number of matched bases and does not report the positions of mismatches, so it cannot be used for sequence alignment solely. Besides, the algorithm does not handle insert and deletion. However, the average indel rate of Illumina library is $10^{-6}$ to $10^{-5}$ [16], and the low indel rate is almost negligible in real data analysis. In addition, Atria does four pairs of matches in 
477 different locations to compensate for the limitation. If one match is failed because of

478 indel, other matches will suggest the real adapter positions.

479 In the runtime benchmark, we compared how trimmers performed using extremely

480 high CPU cores. In general, efficiency marginally decreased as CPU usage increased 481 due to the trimmers' parallel implementation and the inevitable cost of multi-threading, 482 such as task scheduling and context switching. In addition, IO could be the main 483 bottleneck for most hard disk drives and some solid-state drives. Thus, if the system IO 484 reaches a bottleneck, an efficiency plateau would be expected sooner.

\section{Conclusions}

487 We introduce not only Atria, a cutting-edge trimming software for sequence data, but 488 also the ultra-fast and lightweight byte-based matching algorithm. The algorithm can 489 be used in a broad range of short-sequence matching applications, such as primer search 490 and seed scanning before alignment. Atria is implemented in Julia, a programming 491 language designed specifically for high performance. The source code, executables, and 492 benchmark scripts are available on Atria's Github page [24].

\section{Availability and requirements}

495 Project name: Atria

496 Project home page: https://github.com/cihga39871/Atria

497 Operating system(s): Linux, OSX 
498 Programming language: Julia

499 Other requirements: Julia v1.4, Pigz v2.4 or higher, Pbzip v1.1.13 or higher

500 License: MIT

501 Research Resource Identification Initiative ID: SCR_021313

503 Data Availability

504 The datasets SRR330569, and ERR4695159 analyzed during the current study are

505 available in the Sequence Read Archive from the National Center for Biotechnology

506 Information [11, 25].

507 The Atria source codes, releases, documents, and benchmark scripts can be 508 downloaded from Atria's Github page [24].

509

\section{Abbreviations}

511 CPU: Central processing unit; DNA: Deoxyribonucleic acid; GB: Gigabyte; MCC:

512 Matthew's correlation coefficient; NGS: Next-generation sequencing; PPV: Positive

513 predictive value; RAM: Random-access memory; RNA: Ribonucleic acid; SNP: Single

514 nucleotide polymorphism; SSD: Solid-state drive; TB: Terabyte; UInt: Unsigned

515 integer; UInt64: Unsigned 64-bit integer; WGS: Whole-genome sequencing.

\section{Competing interests}

518 The authors declare that they have no competing interests. 


\section{$520 \quad$ Funding}

521 This study was partially funded by the Interdepartmental funding of Genomics

522 Research and Development Initiatives (GRDI), Canada to XL.

\section{Authors' contributions}

525 JC developed Atria software, performed benchmark experiments under the supervision

526 of XL. Both XL and LH serve as co-supervisors and participates in the design of the

527 study. $\mathrm{MH}$ contributes to the optimization of the algorithm. AZ participates in

528 benchmark validation. JC, LH, and XL drafted the manuscript. All authors read and 529 approved the final version of the manuscript.

\section{Acknowledgments}

532 The technical assistance of Jingbai Nie is greatly acknowledged. The financial support

533 of CFIA to JC is greatly appreciated. The advice on algorithm optimization,

534 encouragement, and support of Drs. Christian Lacroix and Stevan Springer to JC are

535 greatly appreciated.

\section{References}

538 1. Schluth-Bolard C, Diguet F, Chatron N, Rollat-Farnier PA, Bardel C, Afenjar 539 A, et al. Whole genome paired-end sequencing elucidates functional and 540 phenotypic consequences of balanced chromosomal rearrangement in patients 
with developmental disorders. J Med Genet. 2019;56 8:526-35. doi:10.1136/jmedgenet-2018-105778.

2. Tan G, Opitz L, Schlapbach R and Rehrauer H. Long fragments achieve lower base quality in Illumina paired-end sequencing. Sci Rep. 2019;9 1:2856. doi:10.1038/s41598-019-39076-7.

4. Krueger F. Trim galore. A wrapper tool around Cutadapt and FastQC to

5. Bolger AM, Lohse $\mathrm{M}$ and Usadel B. Trimmomatic: a flexible trimmer for

3. Schubert M, Lindgreen S and Orlando L. AdapterRemoval v2: rapid adapter trimming, identification, and read merging. BMC Res Notes. 2016;9 1:88. doi:10.1186/s13104-016-1900-2.

10. Jiang H, Lei R, Ding SW and Zhu S. Skewer: a fast and accurate adapter trimmer Illumina sequence data. Bioinformatics. 2014;30 15:2114-20. doi:10.1093/bioinformatics/btu170.

6. BioJulia/BioSequences.jl: Biological sequences for the Julia language. https://github.com/BioJulia/BioSequences.jl. Accessed 1 Dec 2020.

7. Pigz - Parallel gzip. https://zlib.net/pigz/. Accessed 1 Dec 2020.

8. $\quad$ Parallel BZIP2 (PBZIP2). http://compression.ca/pbzip2/. Accessed 1 Feb 2021.

9. Huang W, Li L, Myers JR and Marth GT. ART: a next-generation sequencing read simulator. Bioinformatics. 2012;28 4:593-4. doi:10.1093/bioinformatics/btr708. for next-generation sequencing paired-end reads. BMC Bioinformatics. 2014;15 1:182. doi:10.1186/1471-2105-15-182.

11. Barrett T, Clark K, Gevorgyan R, Gorelenkov V, Gribov E, Karsch-Mizrachi I, et al. BioProject and BioSample databases at NCBI: facilitating capture and organization of metadata. Nucleic Acids Res. 2012;40 D1:D57-D63.

12. Chen S, Zhou Y, Chen Y and Gu J. fastp: an ultra-fast all-in-one FASTQ $\begin{array}{lll}\text { preprocessor. } & \text { Bioinformatics. }\end{array}$ doi:10.1093/bioinformatics/bty560.

13. Sun K. Ktrim: an extra-fast and accurate adapter- and quality-trimmer for sequencing data. Bioinformatics. 2020;36 11:3561-2. doi:10.1093/bioinformatics/btaa171.

14. Didion JP, Martin M and Collins FS. Atropos: specific, sensitive, and speedy trimming of sequencing reads. PeerJ. 2017;5:e3720. doi:10.7717/peerj.3720.

15. Sturm M, Schroeder C and Bauer P. SeqPurge: highly-sensitive adapter trimming for paired-end NGS data. BMC Bioinformatics. 2016;17 1:208. doi:10.1186/s12859-016-1069-7.

16. Schirmer M, D'Amore R, Ijaz UZ, Hall N and Quince C. Illumina error profiles: resolving fine-scale variation in metagenomic sequencing data. BMC Bioinformatics. 2016;17 1:125. doi:10.1186/s12859-016-0976-y. 
581

582

583

584

585

586

587

588

589

590

591

592

593

594

595

596

597

598

599

600

601

602

603

604

605

606

607

608 Figure legends

609

610

611

612

613

614

17. Thurmond J, Goodman JL, Strelets VB, Attrill H, Gramates LS, Marygold SJ, et al. FlyBase 2.0: the next generation. Nucleic Acids Res. 2019;47 D1:D759D65. doi:10.1093/nar/gky1003.

18. Kim D, Paggi JM, Park C, Bennett C and Salzberg SL. Graph-based genome alignment and genotyping with HISAT2 and HISAT-genotype. Nat Biotechnol. 2019;37 8:907-15. doi:10.1038/s41587-019-0201-4.

19. Li H, Handsaker B, Wysoker A, Fennell T, Ruan J, Homer N, et al. The Sequence Alignment/Map format and SAMtools. Bioinformatics. 2009;25 16:2078-9. doi:10.1093/bioinformatics/btp352.

20. Sun K, Jiang P, Wong AIC, Cheng YKY, Cheng SH, Zhang H, et al. Size-tagged preferred ends in maternal plasma DNA shed light on the production mechanism and show utility in noninvasive prenatal testing. Proceedings of the National Academy of Sciences. 2018;115 22:E5106-E14.

21. Langmead B, Wilks C, Antonescu V and Charles R. Scaling read aligners to hundreds of threads on general-purpose processors. Bioinformatics. 2019;35 3:421-32. doi:10.1093/bioinformatics/bty648.

22. Needleman SB and Wunsch CD. A general method applicable to the search for similarities in the amino acid sequence of two proteins. J Mol Biol. 1970;48 3:443-53. doi:10.1016/0022-2836(70)90057-4.

23. Smith TF and Waterman MS. Identification of common molecular subsequences. J Mol Biol. 1981;147 1:195-7. doi:10.1016/00222836(81)90087-5.

24. cihga39871/Atria: An ultra-fast and accurate NGS adapter and quality trimmer. https://github.com/cihga39871/Atria. Accessed 31 Mar 2021.

25. Sequence Read Archive from the National Center for Biotechnology Information. https://www.ncbi.nlm.nih.gov/sra/. Accessed 15 Jan 2021.

\section{Figure 1 Overview of Atria workflow}

\section{Figure 2 Adapter trimming algorithms}

\section{Figure 3 Benchmark of adapter-trimming speed for uncompressed and}

\section{compressed files on different threading options}

The $8.9 \mathrm{G}$ bases simulated paired-end data with a 100 bp read length was trimmed in both uncompressed and compressed format using up to 32 threads. Speed is the ratio of 
615 the number of bases to elapsed time (wall time). SeqPurge does not support

616 uncompressed outputs, so it is not shown in the uncompressed benchmark. In the

617 trimming for compressed data, the speed of AdapterRemoval, Skewer, Fastp, Atropos,

618 and Trimmomatic kept constant when the number of threads increased from 4 to 32, so

619 we only benchmark those trimmers using 1, 2, and 4 threads. Ktrim does not support

620 output compressed files, so it is not shown in the compressed benchmark.

621 Figure 4 Adapter trimming accuracy on adapter presence and absence, different

622 base errors, and adapter lengths

623 A1, B1, and C1 are statistics for reads with adapter contamination, while A2, B2, C2

624 for reads without adapters. A1 and A2 show the accumulated rates of accurate trim, one

625 bp over trim, one bp under trim, multiple bp over trim, and multiple bp under trim. In

626 A1, the accuracy of Trimmomatic is $41.0 \%$. In A2, the accuracy of SeqPurge is $78.8 \%$,

627 the accuracy of Trim Galore is $68.3 \%$. B1 and B2 show the trimming accuracy on

628 different error profiles. In B1, the accuracy of Trimmomatic drops from $41.9 \%$ to

$62940.1 \%$. In B2, the accuracy of SeqPurge is 78.8\%, and the accuracy of Trim Galore is

$63068.2-68.3 \%$. C1 and C2 show the trimming accuracy on different adapter lengths. In

$631 \mathrm{C} 1$, the accuracy of Trimmomatic is $0.0 \%$ at 16 bp adapter length, $50.7 \%$ to $51.6 \%$ at

632 adapter lengths from 20 to $33 \mathrm{bp}$. In C2, the accuracy of SeqPurge ranges from 78.7\%

633 at 16 bp to $78.9 \%$ at 33 bp, and the accuracy of Trim Galore ranges in $68.2-68.3 \%$

634 from 16 to $33 \mathrm{bp}$. 


\section{Supplementary material}

637 Table S1 Trimming speed on the $8.9 \mathrm{G}$ bases $100 \mathrm{bp}$ paired-end simulated data

638 Atria (consensus) does both adapter trimming and paired-end consensus call (base

639 correction of overlapped regions). In the trimming for uncompressed data, SeqPurge

640 does not support uncompressed outputs, so it is not shown in the uncompressed

641 benchmark. Fastp does not support 32 threads, so only 1-16 threads were tested. In the

642 trimming for compressed data, the speed of AdapterRemoval, Skewer, Fastp, and

643 Trimmomatic kept constant when the number of threads increased from 4 to 32, so we

644 only benchmarked those trimmers using 1, 2, and 4 threads. Atropos was too slow to

645 trim compressed data, and Ktrim did not support compressed outputs, so they are not

646 shown in the compressed benchmark.

647

648

649

650

651

652 Table 2 Performance of trimmers on real data (larger than A4)

\begin{tabular}{|c|c|c|c|c|c|c|c|c|c|c|}
\hline \multirow[b]{4}{*}{ Metric } & \multicolumn{2}{|c|}{$\begin{array}{c}\text { Trimming and } \\
\text { consensus }\end{array}$} & \multicolumn{8}{|c|}{ Trimming only } \\
\hline & \multirow[b]{3}{*}{ Atria } & \multirow{3}{*}{$\begin{array}{l}\text { Skewe } \\
\text { r }\end{array}$} & \multirow[b]{3}{*}{ Atria } & \multirow[b]{3}{*}{ AR } & \multirow{3}{*}{$\begin{array}{l}\text { Atrop } \\
\text { os }\end{array}$} & \multirow[b]{3}{*}{ Fastp } & \multirow{3}{*}{$\begin{array}{l}\text { Ktrim } \\
*\end{array}$} & \multirow{3}{*}{$\begin{array}{l}\text { SeqPu } \\
\text { rge }\end{array}$} & \multirow{3}{*}{$\begin{array}{l}\text { Trim } \\
\text { Galor } \\
\text { e }\end{array}$} & \multirow{3}{*}{$\begin{array}{l}\text { Trim } \\
\text { moma } \\
\text { tic }\end{array}$} \\
\hline & & & & & & & & & & \\
\hline & & & & & & & & & & \\
\hline \multicolumn{11}{|c|}{ Low-quality dataset (SRR330569, RNA, Hisat2 mapping) } \\
\hline Elapsed time & & & & & & & $1: 34+$ & & & \\
\hline$(\min : \mathrm{sec})^{*}$ & 2:38 & 9:19 & $2: 32$ & 11:29 & 10:08 & 9:17 & GZ & 3:53 & $\underline{3: 39}$ & 9:38 \\
\hline
\end{tabular}




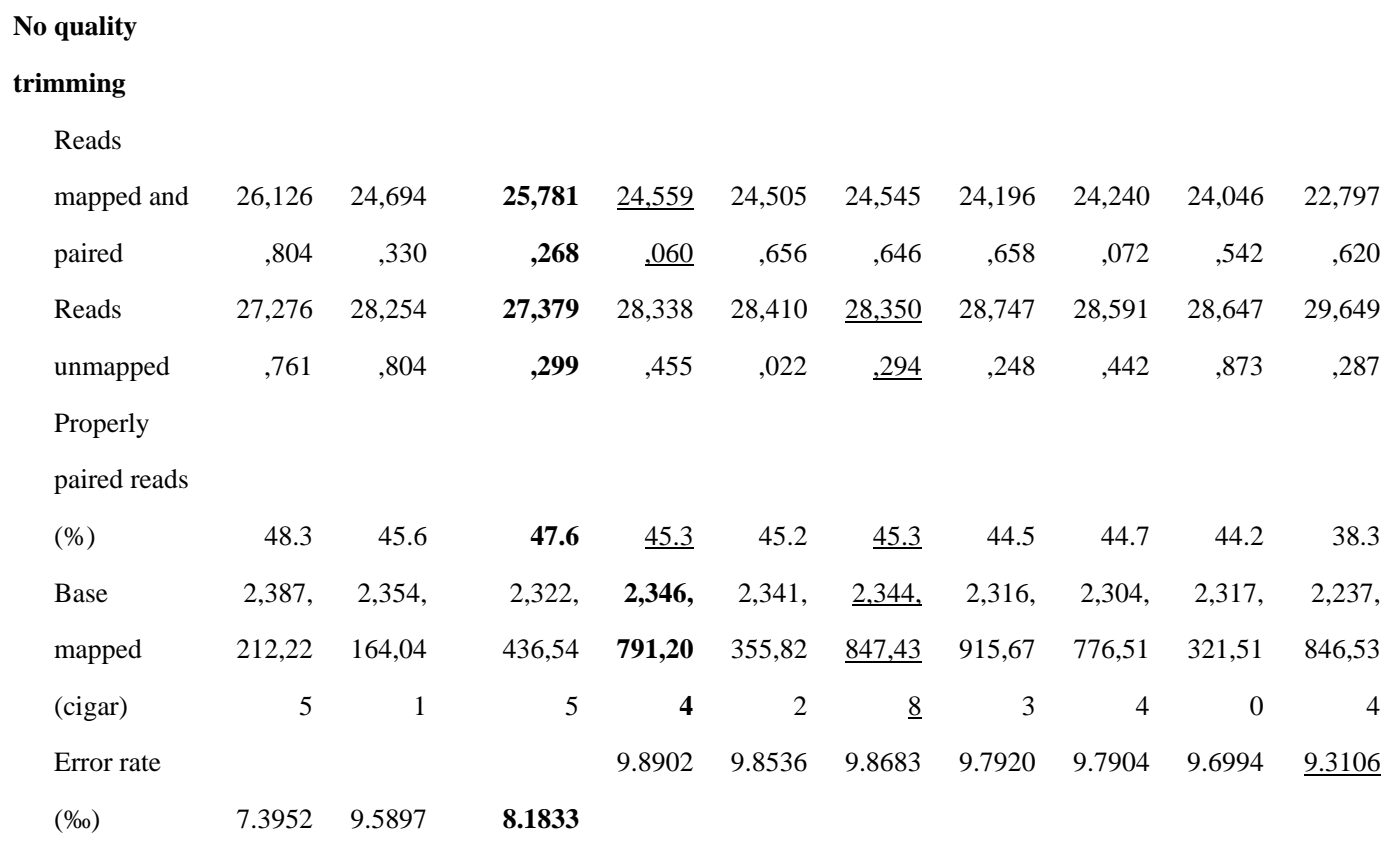

With quality trimming

Reads

\begin{tabular}{|c|c|c|c|c|c|c|c|c|c|c|}
\hline mapped and & 25,942 & 25,787 & 25,728 & 25,721 & 25,714 & $\underline{25,725}$ & 25,473 & 25,364 & 25,654 & 24,744 \\
\hline paired & ,092 & ,464 & ,206 & ,788 & ,956 &, 480 & ,670 & 392 & ,498 & ,754 \\
\hline Reads & 27,245 & 27,364 & 27,361 & 27,369 & 27,373 & $\underline{27,360}$ & 27,556 & 27,736 & 27,400 & 28,064 \\
\hline unmapped & ,720 & ,827 & ,655 & ,773 & ,854 &, 527 & ,820 & ,292 & 932 & ,739 \\
\hline \multicolumn{11}{|l|}{ Properly } \\
\hline \multicolumn{11}{|l|}{ paired reads } \\
\hline (\%) & 47.9 & 47.6 & 47.5 & 47.5 & 47.5 & 47.5 & 46.9 & 46.8 & 47.3 & 42.3 \\
\hline Base & 2,317 & 2,316 & 2,302 , & 2,304 & 2,304 & $\underline{2,304}$, & 2,292 , & 2,263 & 2,297 & 2,246, \\
\hline mapped & 238,53 & 981,45 & 740,46 & 584,26 & 325,74 & $\underline{437,24}$ & 034,76 & 465,11 & 815,43 & 076,61 \\
\hline (cigar) & 6 & 6 & 3 & 9 & 3 & $\underline{4}$ & 2 & 0 & 9 & 8 \\
\hline Error rate & & & & 7.9160 & 7.9141 & 7.9149 & 7.9059 & 7.8787 & 7.8921 & 7.56 \\
\hline
\end{tabular}

(\%) $\quad 7.1114 \quad 7.7882 \quad \underline{7.8902}$

High-quality dataset (ERR4695159, cell-free DNA, Bowtie2 mapping)

Elapsed time

(min:sec)*

3:03

13:48

$13: 41$

$1: 41+$

No quality trimming

Reads

\begin{tabular}{|c|c|c|c|c|c|c|c|c|c|c|}
\hline mapped and & 54,367 & 54,287 & $\underline{54,324}$ & 54,319 & 54,299 & 54,322 & 53,087 & 54,446 & 54,218 & 54,128 \\
\hline paired & ,548 & ,616 &, 964 & ,438 & 922 & 088 & ,420 & 104 & 344 & ,760 \\
\hline Reads & 1,094, & 1,016, & 1,119, & $\underline{989,33}$ & 1,002, & 978,66 & 2,317, & 999,09 & 1,041, & 1,094, \\
\hline unmapped & 145 & 244 & 103 & $\underline{5}$ & 745 & 5 & 968 & 9 & 005 & 752 \\
\hline \multicolumn{11}{|l|}{ Properly } \\
\hline \multicolumn{11}{|l|}{ paired reads } \\
\hline (\%) & 96.8 & 96.7 & 96.7 & $\underline{96.7}$ & $\underline{96.7}$ & 96.7 & 94.1 & 97.0 & 96.4 & 8 \\
\hline
\end{tabular}




$\begin{array}{lrrrrrrrrrr}\text { Base } & 7,703, & 7,700, & 7,700, & \underline{7,701,} & 7,700, & 7,699, & 7,607, & 7,512, & 7,677, & \mathbf{7 , 7 2 0 ,} \\ \text { mapped } & 820,58 & 134,67 & 298,21 & \underline{482,30} & 749,16 & 298,00 & 799,30 & 839,36 & 087,84 & \mathbf{3 5 2 , 4 9} \\ \text { (cigar) } & 5 & 3 & 7 & \underline{2} & 4 & 8 & 6 & 0 & 5 & \mathbf{3} \\ \text { Error rate } & & & & 3.8771 & 3.8834 & \underline{3.8564} & 4.3239 & \mathbf{3 . 8 0 0 7} & 3.9173 & 6.1984 \\ (\%) & 3.3082 & 3.8388 & 3.8724 & & & & & & & \end{array}$

\section{With quality trimming}

\begin{tabular}{lrrrrrrrrrr} 
Reads & & & & & & & & & & \\
mapped and & 54,553 & 54,526 & 54,546 & 54,541 & 54,539 & $\underline{54,549}$ & 53,335 & $\mathbf{5 4 , 6 0 8}$ & 54,482 & 54,403 \\
paired &, 566 &, 276 &, 192 &, 948 &, 502 & $\underline{462}$ &, 674 & $\mathbf{3 0 8}$ &, 002 &, 982 \\
& & & & & & & & & & \\
Reads & 965,44 & 984,84 & 967,91 & 970,86 & 973,21 & $\mathbf{8 2 6 , 4 2}$ & 2,136, & $\underline{890,88}$ & 999,91 & 914,00 \\
unmapped & 7 & 5 & 7 & 9 & 7 & $\mathbf{4}$ & 081 & $\underline{4}$ & 8 & 3 \\
Properly & & & & & & & & & & \\
paired reads & & & & & & & & & & \\
(\%) & 97.0 & 97.0 & $\underline{97.0}$ & $\underline{97.0}$ & $\underline{97.0}$ & $\underline{97.0}$ & 94.4 & $\mathbf{9 7 . 1}$ & 96.8 & 89.0 \\
Base & 7,653, & 7,649, & 7,646, & 7,647, & $\underline{7,648,}$ & 7,646, & 7,556, & 7,461, & 7,625, & $\mathbf{7 , 6 6 8 ,}$ \\
mapped & 879,31 & 380,21 & 989,36 & 893,62 & $\underline{184,19}$ & 574,60 & 468,88 & 588,48 & 484,70 & $\mathbf{7 7 7 , 9 7}$ \\
(cigar) & 2 & 8 & 2 & 4 & $\underline{6}$ & 6 & 2 & 2 & 6 & $\mathbf{1}$ \\
Error rate & & & & 3.2698 & 3.2792 & $\underline{3.2634}$ & 3.7183 & $\mathbf{3 . 2 1 1 7}$ & 3.3109 & 5.5798 \\
(\%) & 2.9547 & 3.2535 & 3.2678 & & & & & & & \\
\hline
\end{tabular}




\section{Adapter} Trimming

\section{Consensus Calling of} Overlapped Region

|ШШШ|Ш|Ш|Ш||

ADAPTER2

\section{READ1 INSERT}

\section{Quality}

Trimming
READ1 INSERT

|||||||||||||||||||||||||||||||||||||||||||||||||||||||||||||||||||||||||||||||||||||||||||||||||||||||||||||

READ2 INSERT 
Encoding a DNA sequence to a dense array

\section{A}

DNA Encoding

\section{C}

Matching \& Scoring (cont.) prevent from overflow

Extracting a 16-mer subsequence as a 64-bit unsigned integer (UInt64)

Extract from memory position 0 (16 bases extracted, sequence indices [0:15], valid indices [0:10])
$00010010010010001111000100100100100011111000 \quad 0000 \quad 0000 \quad 0000 \quad 0000 \quad 0000$

8-bit

Extract from memory position 1 ( 16 bases extracted, sequence indices [2:17], valid indices [2:10])

8 bits ( 1 byte, 2 bases) is the smallest addressable unit of memory in many computer architectures

B

\section{Matching} Algorithm

Given two sequences $a$ and $b$, matching the head of a to each memory position of $b$

D
Decision
Rules

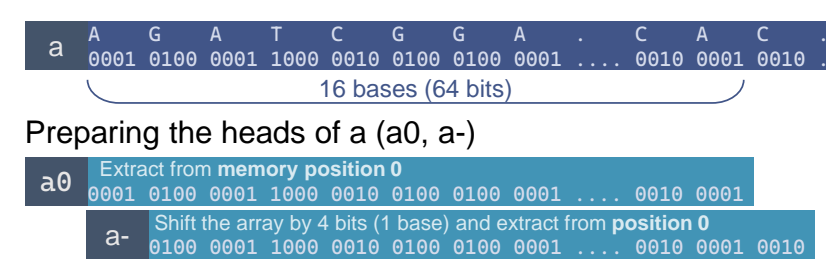

Matching (a0, a-) to each memory position of $b$, and the best index of $b$ is returned

$\begin{array}{lllllllllllllllllll}b & G & C & C & A & G & G & T & C & G & G & A & & C & A & C & A & - \\ 0 & 0100 & 0010 & 0010 & 0001 & 0100 & 0100 & 1000 & 0010 & 0100 & 0100 & 0001 & \ldots & 0010 & 0001 & 0010 & 0001 & 0000 & \ldots\end{array}$ b: memory position 0 , sequence indices [0:15]

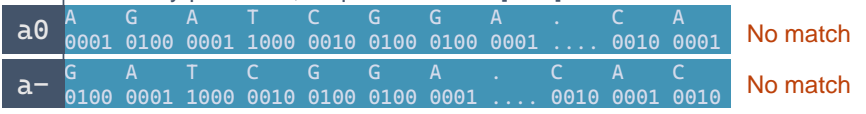

b: memory position 1, sequence indices [2:17]

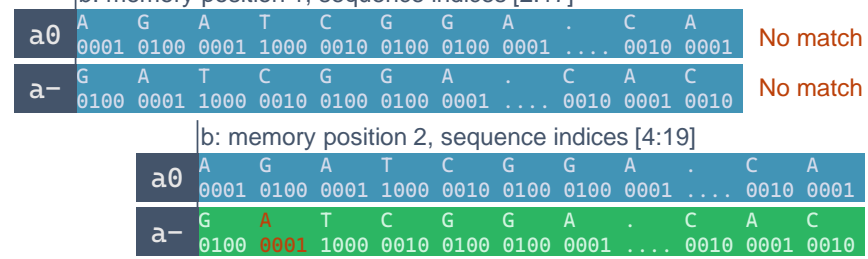

No match

b: memory position 3 , sequence indices [6:21] ... b: memory position $n$,

sequence indices $[2 n: 2 n+15]$
Match (1 error)

\section{C}

Matching \& Scoring

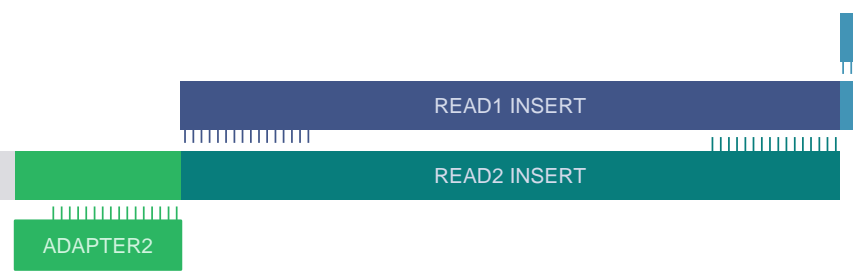

ADAPTER1

ADAPTER1
IIIIIIIIIIII

1) Matching adapter 1 to read 1

Insert size $\mathrm{I}_{\mathrm{A} 1}$, matching score $\mathrm{S}_{\mathrm{A} 1}$

(2) Matching read 1 to reverse complement of read 2 Insert size $\mathbf{I}_{\mathbf{P} 1}$, matching score $\mathbf{S}_{\mathbf{P} 1}$

(3) Matching adapter 2 to read 2 Insert size $\mathrm{I}_{\mathrm{A} 2}$, matching score $\mathrm{S}_{\mathrm{A} 2}$

(4) Matching read 2 to reverse complement of read 1 Insert size $\mathrm{I}_{\mathrm{P} 1}$, matching score $\mathrm{S}_{\mathrm{P} 2}$

Correcting insert size in each read. For $\mathrm{i}$ in 1,2 :

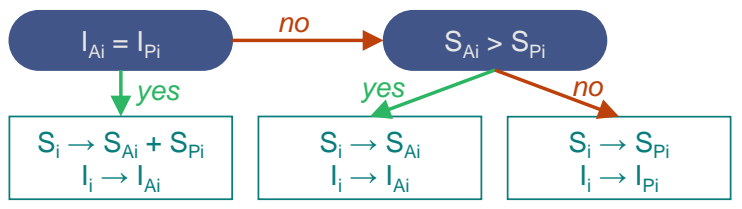

Correcting insert size from its paired read.

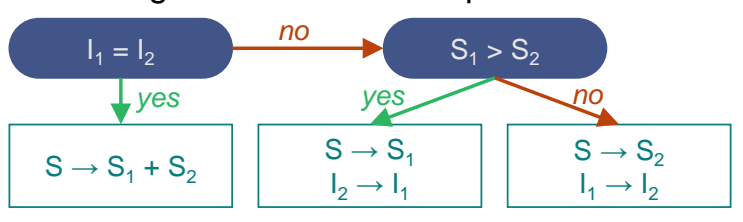

Checking false positive and trim.

$L_{1 / 2}$ as the valid sequence length of read 1 or 2 .

$\mathbf{L}_{\text {tail }}$ as the user-defined tail length.

$\mathbf{E}$ as whether a match is found at the end of read (Bool).

$\mathbf{R}$ as whether the adapter and insert matches suggest

the same insert size (Bool).

$\mathbf{S}_{\text {trim }}$ as the minimum score to trim adapters.

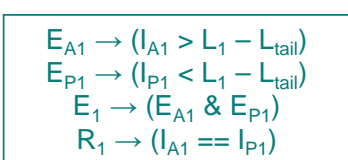

$\begin{aligned} \mathrm{P}_{1} & \rightarrow\left(\mathrm{I}_{\mathrm{P} 1}<\mathrm{L}_{1}-\mathrm{L}_{\text {tai }}\right) \\ \mathrm{E}_{1} & \rightarrow\left(\mathrm{E}_{\mathrm{A} 1} \& \mathrm{E}_{\mathrm{P} 1}\right)\end{aligned}$

$\mathrm{R}_{1} \rightarrow\left(\mathrm{I}_{\mathrm{A} 1}==\mathrm{I}_{\mathrm{P} 1}\right)$

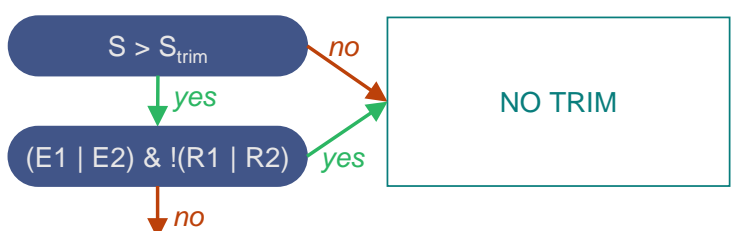

$$
\begin{aligned}
\mathrm{E}_{\mathrm{A} 2} & \rightarrow\left(\mathrm{I}_{\mathrm{A} 2}>\mathrm{L}_{2}-\mathrm{L}_{\text {tail }}\right) \\
\mathrm{E}_{\mathrm{P} 2} & \rightarrow\left(\mathrm{I}_{\mathrm{P} 2}<\mathrm{L}_{2}-\mathrm{L}_{\text {tail }}\right) \\
\mathrm{E}_{2} & \rightarrow\left(\mathrm{E}_{\mathrm{A} 2} \& \mathrm{E}_{\mathrm{P} 2}\right) \\
\mathrm{R}_{2} & \rightarrow\left(\mathrm{I}_{\mathrm{A} 2}==\mathrm{I}_{\mathrm{P} 2}\right)
\end{aligned}
$$

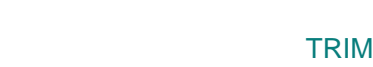



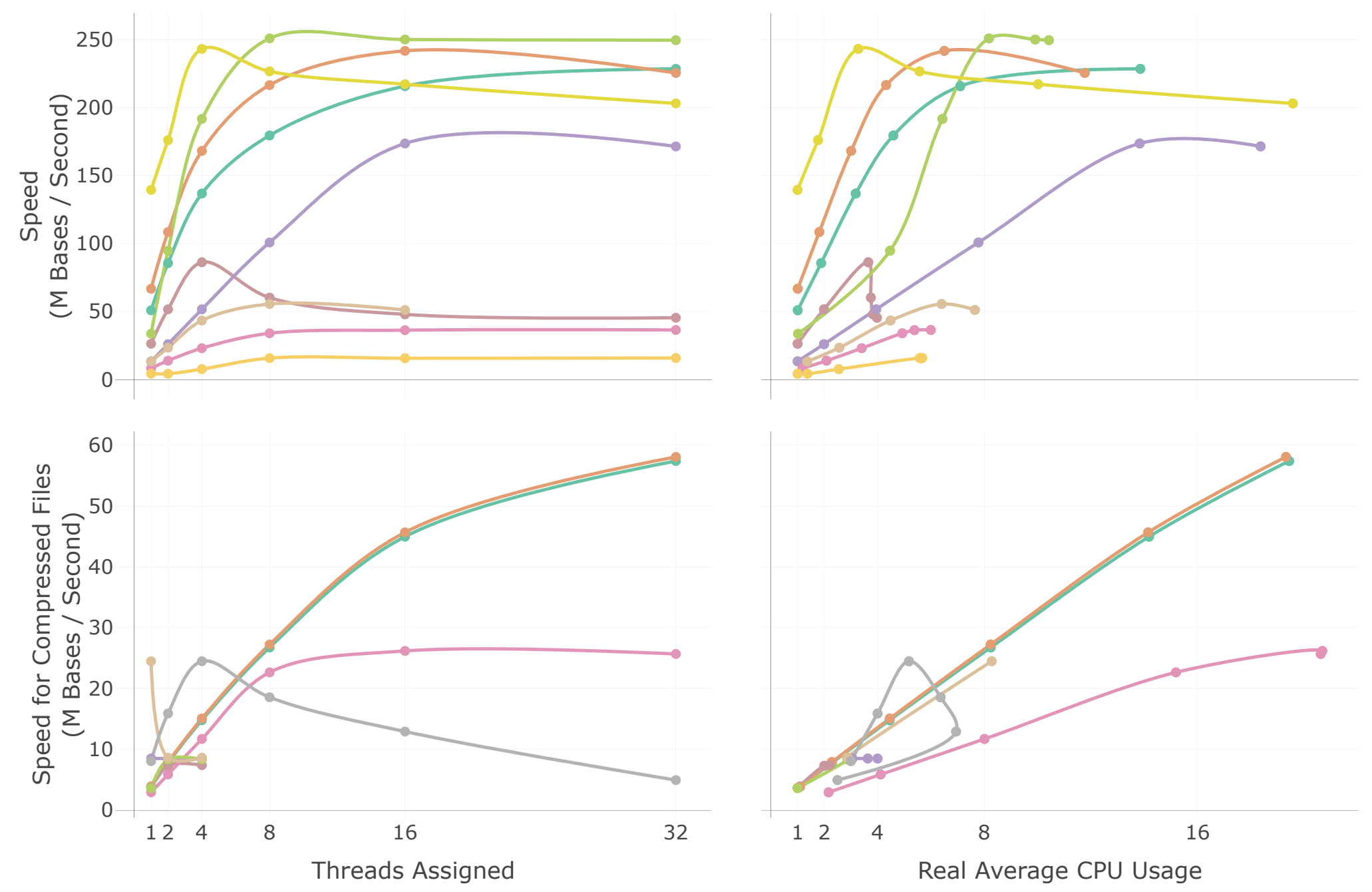


\section{A1}

Reads With Adapter Contamination

(Read Length > Original Insert Size)

\section{A2 Reads Without Adapter Contamination}

AdapterRemoval

Atria

Atropos

Fastp

SeqPurge

Skewe

TrimGalore

Trimmomatic

$96.0 \%$

$97.0 \%$

$98.0 \%$

$99.0 \%$

$100.0 \% 96.0 \%$

$97.0 \%$

$98.0 \%$

Accumulated Rate

B1

$100.0 \%$

B2.

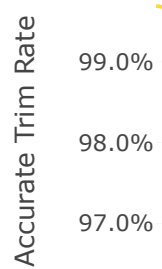

$96.0 \%$

$1 \mathrm{x}$

$2 x$

$3 x$

$4 x$

$5 \mathrm{x} \quad 1 \mathrm{x}$

$2 x$

$3 x$

$4 x$

Read Simulation Error Profile

(Base Rates: Substitution 0.1\%; Insertion 0.001\%; Deletion 0.001\%)

$\mathrm{C} 1$
$100.0 \%$
$99.0 \%$
$98.0 \%$
$97.0 \%$

$\mathrm{C} 2$

$96.0 \%$
Under Trim $>1$ bp Over Trim > 1 bp

Under Trim = $1 \mathrm{bp}$

Over Trim = 1 bp

Accurate Trim

Trimmomatic
TrimGalore
Skewer
SeqPurge
Fastp
Atropos
Atria
AdapterRemoval

